\title{
Microarray analysis of gene expression profiles of cardiac myocytes and fibroblasts after mechanical stress, ionising or ultraviolet radiation
}

\author{
Marjan Boerma* ${ }^{* 1,3}$, Caroline GC van der Wees ${ }^{\dagger 1,2}$, Harry Vrieling1, J \\ Peter Svensson ${ }^{1}$, Jan Wondergem ${ }^{3}$, Arnoud van der Laarse ${ }^{2}$, \\ Leon HF Mullenders ${ }^{1}$ and Albert A van Zeeland ${ }^{1}$
}

\begin{abstract}
Address: ${ }^{1}$ Department of Toxicogenetics, Leiden University Medical Center, Wassenaarseweg 72, 2333 AL Leiden, The Netherlands, ${ }^{2}$ Department of Cardiology, Leiden University Medical Center, Albinusdreef 2, 2333 ZA Leiden, The Netherlands and ${ }^{3}$ Department of Clinical Oncology, Leiden University Medical Center, Albinusdreef 2, 2333 ZA Leiden, The Netherlands

Email: Marjan Boerma* - mboerma@uams.edu; Caroline GC van der Wees - c.g.c.van_der_wees@lumc.nl; Harry Vrieling - h.vrieling@lumc.nl; J Peter Svensson - p.svensson@lumc.nl; Jan Wondergem - j.wondergem@lumc.nl; Arnoud van der Laarse - a.van_der_laarse@lumc.nl;

Leon HF Mullenders - l.mullenders@lumc.nl; Albert A van Zeeland - a.a.van_zeeland@lumc.nl

* Corresponding author †Equal contributors
\end{abstract}

Published: 18 January 2005

BMC Genomics 2005, 6:6 doi:10.1/86/147|-2164-6-6

This article is available from: http://www.biomedcentral.com/I47/-2/64/6/6

(C) 2005 Boerma et al; licensee BioMed Central Ltd.

This is an Open Access article distributed under the terms of the Creative Commons Attribution License (http://creativecommons.org/licenses/by/2.0), which permits unrestricted use, distribution, and reproduction in any medium, provided the original work is properly cited.
Received: 13 July 2004

Accepted: 18 January 2005

\begin{abstract}
Background: During excessive pressure or volume overload, cardiac cells are subjected to increased mechanical stress (MS). We set out to investigate how the stress response of cardiac cells to MS can be compared to genotoxic stresses induced by DNA damaging agents. We chose for this purpose to use ionising radiation (IR), which during mediastinal radiotherapy can result in cardiac tissue remodelling and diminished heart function, and ultraviolet radiation (UV) that in contrast to IR induces high concentrations of DNA replication- and transcription-blocking lesions.
\end{abstract}

Results: Cultures enriched for neonatal rat cardiac myocytes (CM) or fibroblasts were subjected to any one of the three stressors. Affymetrix microarrays, analysed with Linear Modelling on Probe Level, were used to determine gene expression patterns at 24 hours after (the start of) treatment. The numbers of differentially expressed genes after UV were considerably higher than after IR or MS. Remarkably, after all three stressors the predominant gene expression response in CMenriched fractions was up-regulation, while in fibroblasts genes were more frequently downregulated. To investigate the activation or repression of specific cellular pathways, genes present on the array were assigned to 25 groups, based on their biological function. As an example, in the group of cholesterol biosynthesis a significant proportion of genes was up-regulated in CMenriched fractions after MS, but down-regulated after IR or UV.

Conclusion: Gene expression responses after the types of cellular stress investigated (MS, IR or UV) have a high stressor and cell type specificity. 


\section{Background}

The mammalian myocardium contains several cell types, of which the cardiac myocytes (CM) make up most of the heart's mass. Although a small proportion of $\mathrm{CM}$ in the adult myocardium remains mitotic, most $\mathrm{CM}$ lose the capacity to undergo cell division shortly after birth [1]. In the adult heart, approximately $70 \%$ of the cells is represented by non-myocytes, most of which belong to the fibroblast compartment. In this study, we investigate how the stress response of cardiac cells to increased mechanical stress (MS) can be compared to genotoxic stresses induced by two DNA damaging agents, ionising radiation (IR) and ultraviolet radiation (UV).

In cardiac cells, MS is increased during excessive pressure or volume load of the heart, as seen in hypertensive and valvular heart disease. This results in an adaptive growth response leading to structural and functional cardiac changes, including CM hypertrophy and hyperplasia of fibroblasts, to compensate for the increased workload [2]. In vitro cyclic stretch of rat cardiac cells has been shown to be an appropriate model for cellular changes that occur during overload of cardiac muscle in vivo [3]. Mechanical signals may be transferred to the nucleus of cells through integrin receptors, cytoskeletal filaments and nuclear scaffolds [4] and through ion channels, ion exchangers and hormone receptors [5].

Radiation induced heart disease (RIHD) has been recognised as a late adverse effect of thoracic radiotherapy if the heart was situated in the radiation field [6]. IR induces the formation of reactive oxygen species that react with different components of the cell, thereby inducing macromolecular lesions. IR can activate several signal transduction pathways, involving growth factor receptors, death receptors and DNA damage sensing proteins [7]. Primary fibroblasts in culture are known to go into senescence shortly after IR [8], after which terminal differentiation of these cells is induced [9]. Cultures of CM do not demonstrate cell death, nor a loss of function upon a single dose of 10,000 rad ( 100 Gy) [10,11].

The density of DNA damaging events after UVC, which include helix-distorting photolesions, is three orders of magnitude higher than the density of DNA damage that occurs after IR [12] and sufficient to block cellular DNA replication and transcription. Signal transduction after UV is mediated via components of the cellular membrane, involving growth factor receptors, and via DNA damage sensing proteins [7]. UV is known to induce cell cycle arrest and apoptosis in several cell types $[13,14]$.

The aim of the study was to identify and compare differentially expressed genes (up-regulated or down-regulated when compared with untreated controls) in cardiac cells in response to MS, IR or UV. To this purpose, cultures enriched for ventricular $\mathrm{CM}$ or fibroblasts were exposed to one of the three stressors. Differentially expressed genes were identified using Affymetrix GeneChips. Several statistical methods have been developed to analyse Affymetrix gene expression microarrays. We used a method based on Linear Modelling on Probe Level [15] to describe the signal of every perfect match (PM) probe. Because overall changes in the expression of functionally related genes are more informative than the expression pattern of single genes, genes in microarray studies can be assigned to functional groups [16]. In the present study, such an approach was used to classify genes, based on biological function or on the role of a gene product in common intracellular pathways.

\section{Results \\ Accuracy of the linear model}

As an example for the accuracy of the linear model that was used to describe the PM probe signals, Figure 1A shows a dot plot of all PM probe signals as calculated by the linear model, against the actual PM probe signals determined from fibroblasts after UV. These data were used to calculate correlation coefficients $\left(\mathrm{R}^{2}\right)$ between the signals calculated by the model and the actual PM signals obtained. Figure $1 \mathrm{~B}$ shows the distribution of $\mathrm{R}^{2}$ values for fibroblasts after UV. The majority of probe-sets have a correlation coefficient $\geq 0.90$, indicating that the model used fitted the data accurately.

\section{Numbers of differentially expressed genes}

A Series entry (accession number GSE2032) at Gene Expression Omnibus (GEO), a public gene expression database of NCBI [17], gives access to all microarray data generated in this study. Figure 2 represents the numbers of genes with a unique LocusLink ID that were up-regulated or down-regulated $(\mathrm{q}<0.005)$ in $\mathrm{CM}$-enriched cultures/ fractions and cultures of fibroblasts after one of the three stressors. When using these criteria, the numbers of differentially expressed genes (up-regulated or down-regulated) after UV were considerably higher than after IR or MS. After each of the three stressors more genes were up-regulated in CM-enriched cultures/fractions than in cultures of fibroblasts. Conversely, higher numbers of down-regulated genes were determined in fibroblasts. These differences were most pronounced after MS (Figure 2A).

Based on information available at NettAff ${ }^{\mathrm{TM}}$ [18], extended with standard textbooks and recent literature, genes with a unique LocusLink ID were assigned to several functional groups. Subsequently, the status of each gene within a functional group was determined in both cell populations (CM-enriched cultures/fractions and cultures of fibroblasts). Individual probe-sets within these functional groups and their q-value after the three stressors are 


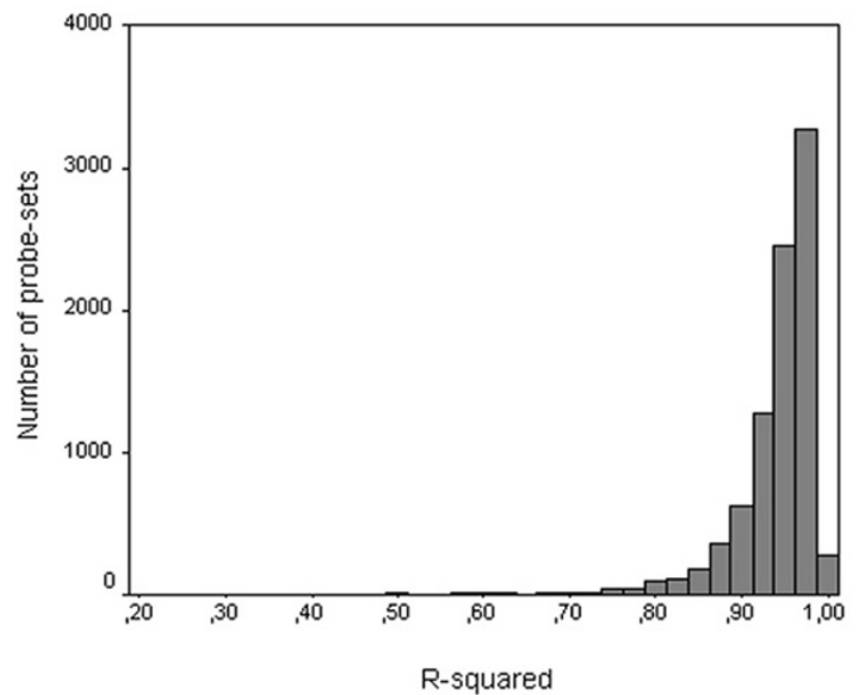

Figure I

Accuracy of the linear model. Dot plot of all PM probe signals as calculated by the linear model, against actual PM probe signals determined from fibroblasts after UV (A). The data presented in Figure IA were used to calculate correlation coefficients $\left(R^{2}\right)$ between the signals calculated by the linear model and the actual PM signals of fibroblasts after UV (B).

listed in table 1 (see additional file 1 ). In this table, downregulated genes are distinguished from up-regulated genes by a minus-sign in front of their q-value. Figure 3 shows the percentage of genes in a functional group that are differentially expressed after MS, IR or UV. In accordance with Figure 2, the highest percentages of genes were differentially expressed after UV (on average 39.4\% for both cell populations), followed by IR (13.0\%). In general, the percentages of differentially expressed genes were lowest after MS (8.3\%). Several functional groups, including heat shock proteins and genes involved in cholesterol biosynthesis, showed high proportions of differentially expressed genes with a hypergeometric probability $\mathrm{P}<$ 0.005 . These functional groups were considered to have significantly high percentages of differentially expressed genes. On the other hand, in the group of genes encoding for ion channels and exchangers, low percentages of differentially expressed genes with a hypergeometric probability $\mathrm{P}<0.005$ were determined, both after IR and UV.

\section{Percentages of up-regulated genes}

Figure 4 shows the percentages of differentially expressed genes that were up-regulated after IR or after UV. In several functional groups, including p53 target genes and genes involved in mitosis, a significant percentage of differentially expressed genes was up-regulated (hypergeometric

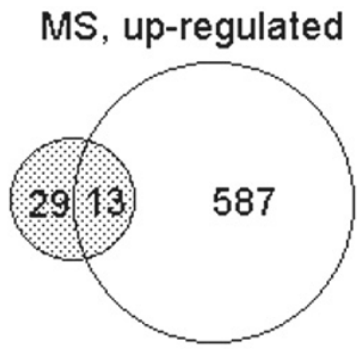

MS, down-regulated

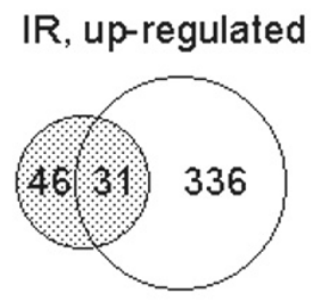

IR, down-regulated

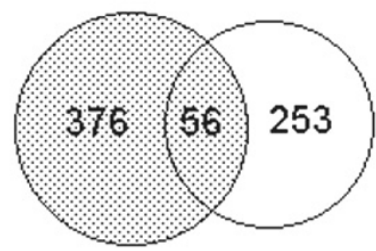

UV, up-regulated

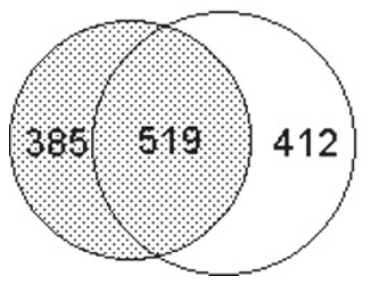

UV, down-regulated

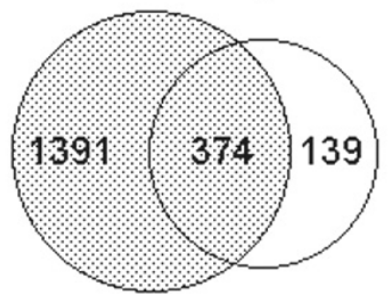

Figure 2

Numbers of differentially expressed genes. Numbers of differentially expressed genes $(q<0.005)$ with a unique LocusLink ID in cultures of fibroblasts (dotted) and CMenriched cultures after MS (A), in cultures of fibroblasts (dotted) and CM-enriched fractions after IR (B), or in cultures of fibroblasts (dotted) and CM-enriched fractions after UV (C). Overlapping parts of the circles represent genes that show differential expression both in CM-enriched cultures/fractions and cultures of fibroblasts.

probability $\mathrm{P}<0.005)$. Other functional groups, including cytoskeletal components and genes involved in cholesterol biosynthesis mainly had down-regulated genes. In all 25 functional groups, the hypergeometric probability $\mathrm{P}$ of the percentage of up-regulated genes after MS was not below the pre-set threshold of 0.005 . Therefore, no significant percentages of up-regulated genes were determined in any of the functional groups after MS (data not shown).

\section{General stress response genes}

Probe-sets that showed an up-regulation or down-regulation after more than one stressor are listed in table 2 (see additional file 2). The overlap in responsive genes between IR and UV was larger than the overlap between 


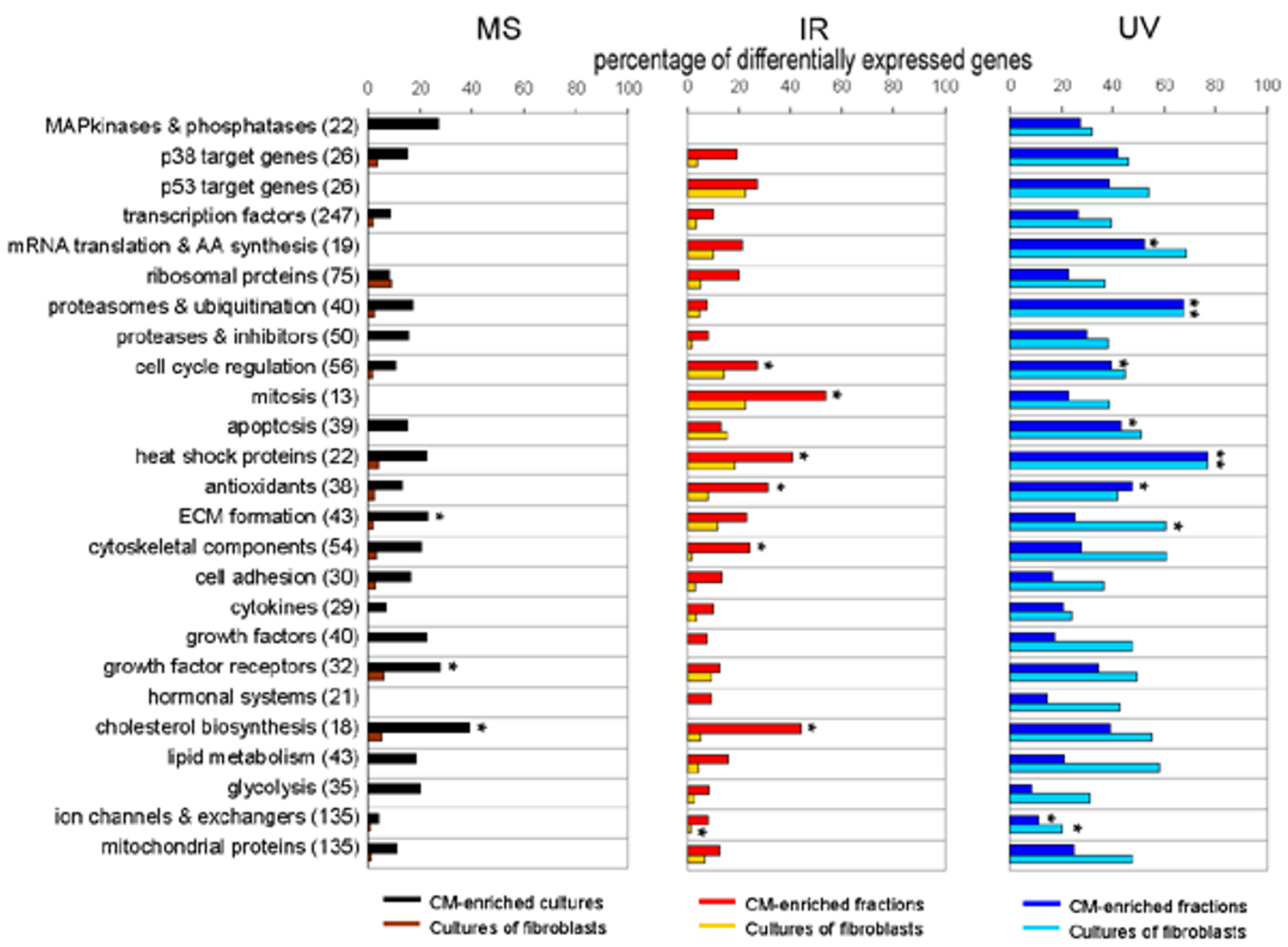

Figure 3

Percentages of differentially expressed genes. Percentage of total number of genes within functional groups that are differentially expressed in CM-enriched cultures/fractions and cultures of fibroblasts after MS, IR or UV. Numbers between brackets represent total numbers of genes within a functional group. For example, of the 22 MAPkinases and phosphatases found to be represented by the array, $27 \%$ were differentially expressed in CM-enriched fractions after UV. AA: amino acid. *Hypergeometric probability $\mathrm{P}<0.005$

MS and one of the radiation types. Both after IR and UV, several genes that are known to play a central role in the radiation response of cells, including p21, GADD153 and mdm2, were up-regulated. These genes were not up-regulated after MS. A striking large proportion of genes encoding cytoskeletal components were down-regulated both after IR and UV.

Validation of microarray results by semi-quantitative $P C R$ Gene expression changes detected using microarrays were validated by semi-quantitative PCR, using RNA from CMenriched cultures at 24 hours after MS. The increased gene expression of Tenascin $\mathrm{C}$ and biglycan were confirmed with PCR in two independent experiments. In these two experiments, Tenascin $\mathrm{C}$ gene expression increased 1.59 and 1.64 times, respectively. Biglycan gene expression increased 1.42 and 1.25 times, respectively. Figure 5 shows a representative result of the Tenascin C PCR.

\section{Discussion}

In this study, a linear model was used to describe GeneChip PM probe signals and to determine effects of three types of stressors on gene expression in two neonatal rat heart cell populations, consisting of CM that are known to be terminally differentiated cells and fibroblasts that still have the capacity to undergo mitosis. Several studies have shown a good correlation between Affymetrix GeneChip data and RT-PCR [16] or Northern-blot [19,20]. In a 


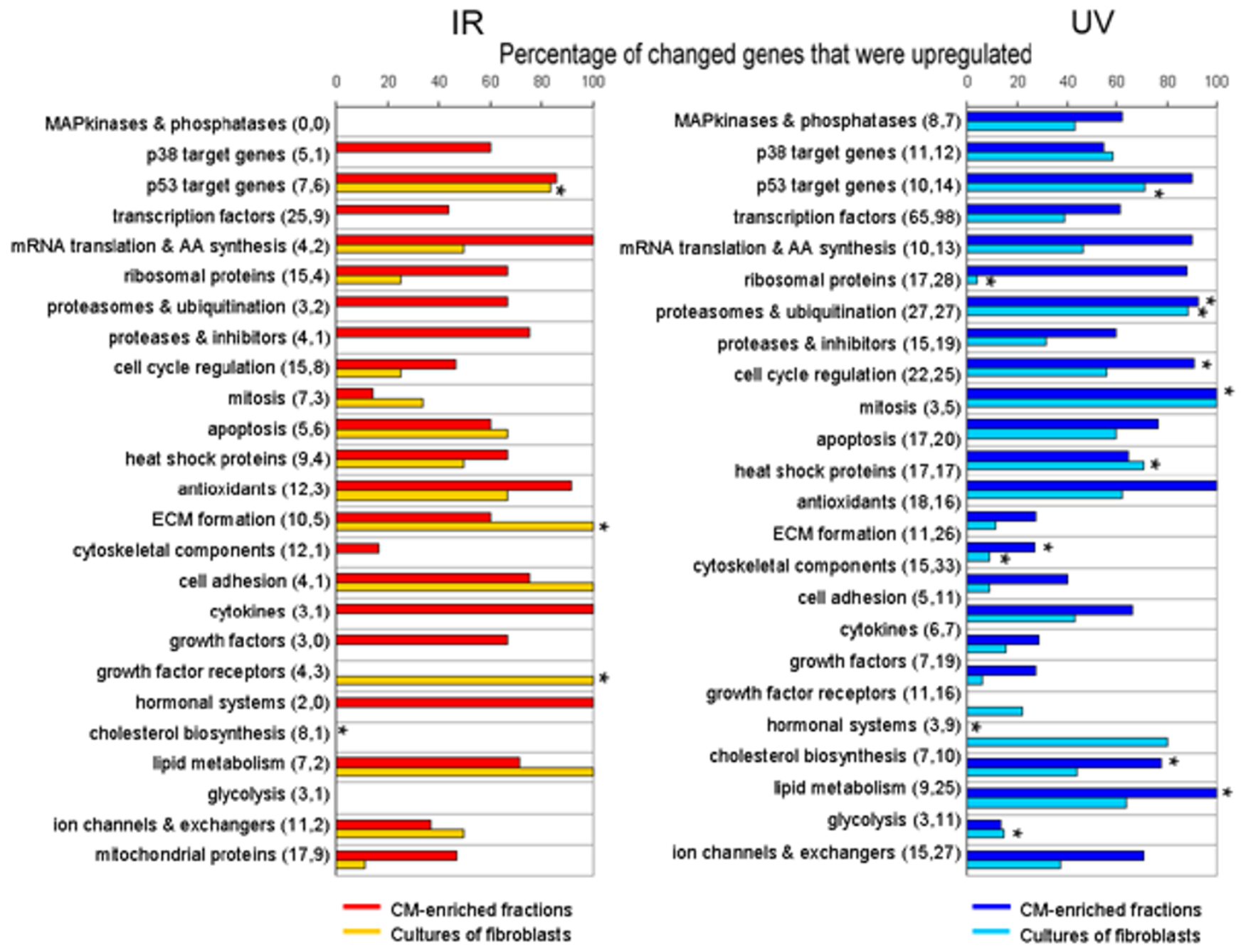

Figure 4

Percentages of up-regulated genes. Percentage of changed genes that were up-regulated per functional group after IR or UV. Numbers between brackets represent numbers of differentially expressed genes in CM-enriched fractions and cultures of fibroblasts, respectively. For example, of the 8 MAPkinases and phosphatases that were differentially expressed in CM-enriched fractions after UV, 63\% were up-regulated. *Hypergeometric probability $\mathrm{P}<0.005$

previous study neonatal rat CM-enriched cultures and cultures of neonatal rat cardiac fibroblasts were irradiated with a single dose of $8.5 \mathrm{~Gy}$ and some mRNA transcripts were quantified by competitive PCR [21]. In accordance with the present study, no significant changes in gene expression of transforming growth factor- $\beta 1$ (TGF- $\beta 1$ ), fibroblast growth factor-2 (FGF-2) and collagen type I were determined at $24 \mathrm{~h}$ after IR in cultures of cardiac fibroblasts. Moreover, no significant IR-induced changes were determined in gene expression of atrial natriuretic peptide (ANP) in CM-enriched cell populations in both the latter and the present study. On the other hand, CMenriched cultures showed a reduced TGF- $\beta 1$ expression (by PCR) at $24 \mathrm{~h}$ after $8.5 \mathrm{~Gy}$, which was not observed in the present study. This might be due to differences in experimental design between the two studies, as in the former study CM-enriched cultures were obtained by incubation with bromodeoxyuridine to prevent fibroblast proliferation. Also, during and after irradiation, the cells were incubated in higher serum concentration than used in the present study.

Here, differences between the two cell populations are observed in the predominant type of gene expression response, i.e. up-regulation versus down-regulation. After all three stressors, differentially expressed genes were 


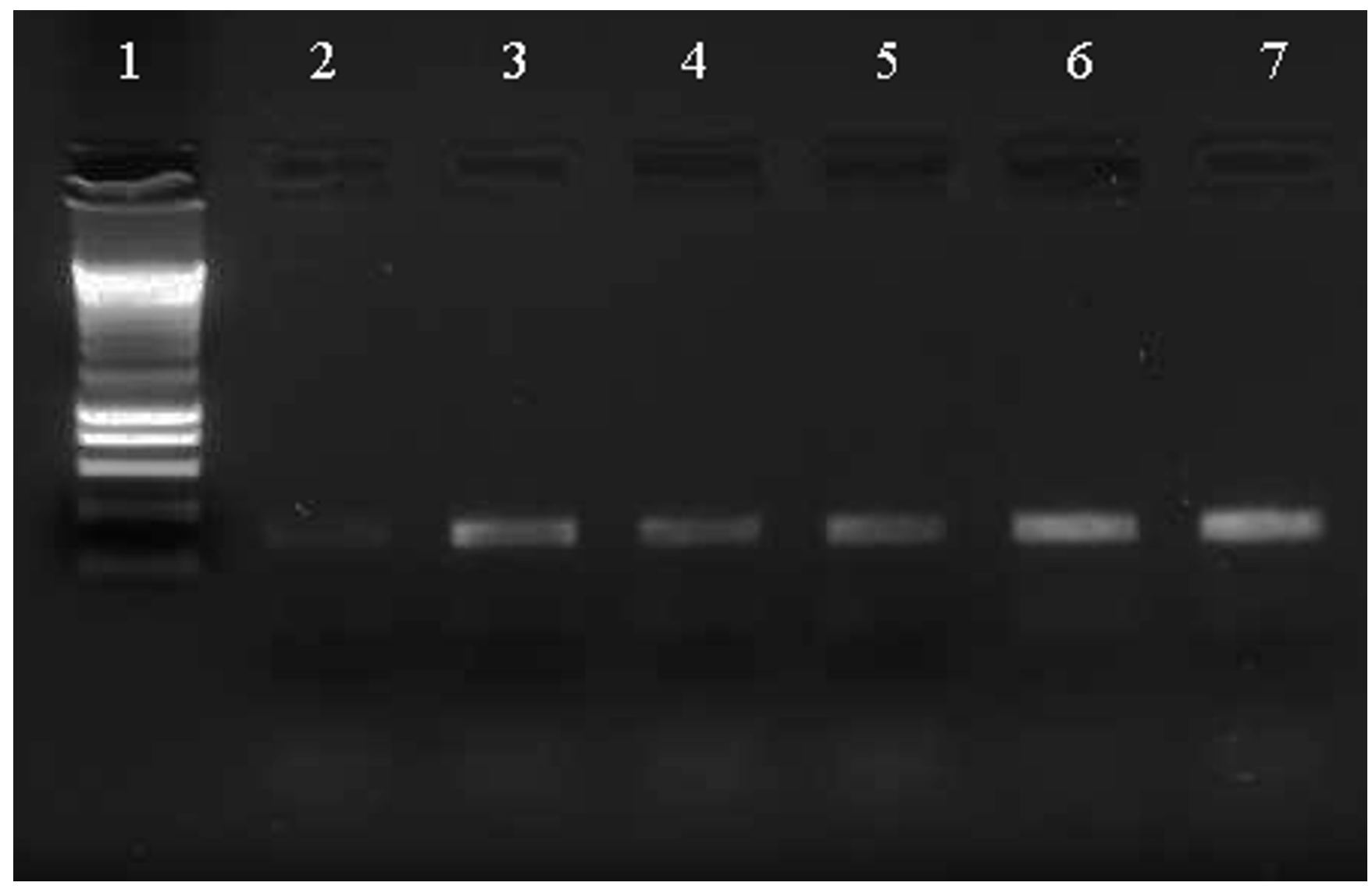

Figure 5

Representative result of the Tenascin C PCR Total RNA was isolated from CM-enriched cultures at 24 hours after MS or control treatment. After cDNA synthesis, semi-quantitative PCR was used to determine Tenascin C gene expression. Lane I: smart ladder; lane 2: negative control; lane 3: positive control; lanes 4 and 5: CM-enriched after control treatment; lanes 6 and 7: CM-enriched after MS.

mostly up-regulated $(\mathrm{q}<0.005)$ in CM-enriched fractions, while in cultures of fibroblasts the majority of changed genes were down-regulated. After MS, these differences were most pronounced. Paracrine signalling is involved in the response of $\mathrm{CM}$ and cardiac fibroblasts in co-cultures subjected to MS [22]. In the CM-enriched cultures used in this study, remaining fibroblasts might stimulate gene transcription of the $\mathrm{CM}$, resulting in higher numbers of up-regulated genes. It cannot be excluded that differences in level of toxicity between the three stressors applied in this study caused differences in gene expression levels.

Of the three stressors examined in this study, both IR and UV are known to induce oxidative stress. Accordingly, both stressors induced high numbers of up-regulated genes involved in anti-oxidative processes. The high percentages of up-regulated genes encoding heat shock proteins in both cell populations after IR and UV might indicate oxidative stress induced protein damage in these cells. In comparison, after MS only few genes encoding heat shock proteins were up-regulated in either cell population.

Heinloth et al. (2003) proposed a model for the regulation of several gene expression patterns after IR and UV in human dermal fibroblasts, based on microarray analysis. The general view that p53 plays a central role in signal transduction after IR and UV was confirmed in their study. Moreover, IR down-regulated the expression of genes involved in mitosis. UV, on the other hand, induced the expression of genes involved in protein degradation and the MAPK pathway [23]. The latter data are in accordance with the data of the present study, showing that UV did affect genes participating in the MAPK pathway (although not significantly) in both cell populations, but IR did not. Moreover, in CM-enriched fractions, IR resulted in a 
down-regulation of a large percentage of genes involved in mitosis. Several genes, involved in cell cycle regulation and mitosis are expressed in foetal cardiac myocytes and down-regulated in adult myocytes, in accordance with their terminal differentiation $[24,25]$. Due to the close relation between foetal and neonatal cells, an expression of mitotic genes in the neonatal CM used in this study can be expected.

The high numbers of up-regulated genes involved in ubiquitination and protein degradation in both cell populations after UV are also in accordance with the study of Heinloth et al. (2003) and with other studies on cultured cells after UV [20]. These results might reflect the need of cells to replace molecules that were damaged by UV irradiation, although the proteasome is also proposed to play a role in several cellular processes, including DNA-repair, cell cycle regulation and cell survival after irradiation [26].

In CM-enriched cultures, MS led to a relatively high proportion of up-regulated genes that are involved in cholesterol biosynthesis. Among these genes, expression of 3-hydroxy-3-methylglutaryl-Coenzyme A reductase, which forms the starting enzyme of the mevalonate pathway and is considered to be the key enzyme in cholesterol biosynthesis, was up-regulated. This suggests that in neonatal rat cardiac myocytes cholesterol biosynthesis is stimulated after MS. Neonatal rat heart myocytes that undergo hypertrophy in culture show an increased biosynthesis and intracellular accumulation of cholesterol [27]. Moreover, the mevalonate pathway has been proposed to play a role in Ras activation in neonatal rat cardiac myocytes subjected to MS in culture, leading to hypertrophy [28]. In vivo hypertrophy of the heart is also associated with elevated myocardial cholesterol contents [29]. An increased cholesterol biosynthesis in cardiac myocytes that undergo MS might therefore accompany a hypertrophic response of these cells. However, of the three foetal genes that are known to be re-expressed in hypertrophic myocytes, i.e. smooth muscle alpha-actin, ANP and beta-myosin heavy chain, only the last gene was up-regulated at the time point of investigation. In contrast to MS, IR and UV led to a down-regulation of genes involved in cholesterol biosynthesis in CM-enriched fractions. In previous studies, alterations in cholesterol contents of cardiac myocytes and fibroblasts were associated with alterations in protein to DNA ratios, levels of several enzymes including ATPases and phosphatases, and diffusion rates of membrane proteins [30-32]. Moreover, a decrease in beating rate observed in aging cultures of CM was opposed by increased membrane amounts of cholesterol [33]. The role of cholesterol biosynthesis in the cellular functions of cardiac cells after MS, IR or UV needs further investigation.
Higher numbers of differentially expressed genes involved in ECM formation, including genes encoding collagens, fibronectin and laminin, were determined in CMenriched cultures after MS than in cultures of fibroblasts. As mentioned before, paracrine signalling between CM and remaining fibroblasts might play a role in cellular responses in these CM-enriched cultures. Alterations in expression of genes involved in ECM formation might originate from remaining fibroblasts after stimulation by $\mathrm{CM}$. Interestingly, MS does not affect the expression of these genes in cultures of fibroblasts, which suggests that paracrine signalling from CM is necessary for alterations in expression profiles of genes involved in ECM formation in fibroblasts.

The down-regulated genes encoding cytoskeletal components in cultures of fibroblasts after IR and UV, including myosin and troponin, are mostly CM-specific. Therefore, these genes are likely to originate from remaining $\mathrm{CM}$ in the fibroblast cultures, although their numbers were low $(3-5 \%)$. The extremely low numbers of differentially expressed genes encoding for ion channels and exchangers might be explained by a low number of cardiac cell type-specific genes within this functional group.

\section{Conclusions}

MS, IR and UV mainly induce stress-specific and cell-type specific gene expression profiles in neonatal rat $\mathrm{CM}$ enriched cultures and cultures of neonatal rat heart fibroblasts. Functional groups that show significant percentages of differentially expressed genes suggest that certain cellular pathways are activated after one or more stresses.

\section{Methods \\ Cell culturing}

The experiments were performed with permission of the local committee on animal experiments, installed by the University of Leiden according to the Dutch law. Cardiac cells were isolated from ventricles of neonatal rat hearts, and cultured as described before [14,34]. By applying a pre-plating method, cultures enriched for $\mathrm{CM}$ and cultures of fibroblasts were obtained.

After 2 days of culturing with 5\% horse serum (HS), culture media of CM-enriched cultures were replaced by media containing $2.5 \%$ HS. One day later, these cultures were subjected to UV, IR or MS.

Three days after isolation, cultures of fibroblasts were subcultured for 6 days in medium containing 10\% foetal bovine serum (FBS). Then, culture medium was replaced by medium containing $2.5 \%$ FBS. One day later, confluent cell cultures were subjected to UV, IR or MS. 


\section{Irradiation and cyclic stretch}

Cells were subjected to a single dose of 8.5 Gy of X-rays at room temperature, using a $6 \mathrm{MV}$ accelerator (SL 75-5 Philips), operated at a dose rate of $8 \mathrm{~Gy} \mathrm{~min}^{-1}$. During irradiation, sham-irradiated control cells were kept at room temperature. After irradiation, cell cultures were maintained at $37^{\circ} \mathrm{C}$ for $24 \mathrm{~h}$.

In another experiment cells were irradiated with $10 \mathrm{~J} / \mathrm{m}^{2}$ UVC at room temperature at a dose rate of $0.2 \mathrm{~J} / \mathrm{m}^{2}$ per s. Before irradiation, medium was collected and cells were rinsed with PBS. Following irradiation, growth medium was returned to the cultures and the cells were incubated for an additional $24 \mathrm{~h}$. Control cells received a similar treatment except for UV irradiation.

To subject cells to cyclic stretch, CM-enriched cells (54 \pm $5 \% \mathrm{CM})$ and fibroblasts (95 $\pm 2 \%$ non-myocytes) were cultured in 6-well Flex I culture plates (Flexcell, Hillsborough, NC, USA), coated with collagen I. Medium was changed to medium containing $2.5 \%$ serum and after $24 \mathrm{~h}$ plates were placed in the Flexercell Strain Unit FX$2000^{\circledast}$ (Flexcell) in which the frequency and magnitude of stretch were regulated by a computer-controlled vacuum pump. The apparatus applied an equiaxial cyclic stretch of $20 \%$ elongation to the wells at a frequency of 60 cycles/ min $(1 \mathrm{~Hz})$. Stretch was applied for $24 \mathrm{~h}$. Control cells were grown in identical culture plates and incubated in the same incubator as the stretched cultures, but were not mounted in the Flexercell Strain Unit.

\section{Cell harvesting after irradiation}

Twenty-four hours after IR or UV, non-irradiated and irradiated CM-enriched cultures were trypsinised and subjected to centrifugal elutriation [35]. The proportion of $\mathrm{CM}$ in each elutriation fraction was determined by flow cytometric analysis of myosin expression, as described before [35]. After elutriation of IR-exposed cultures, CMenriched fractions contained $74 \pm 3 \% \mathrm{CM}$ and after elutriation of UV-exposed cultures, CM-enriched fractions contained $85 \pm 3 \% \mathrm{CM}$. Because of the high purity of the fibroblast cultures (95 $\pm 2 \%$ non-myocytes), no centrifugal elutriation was applied on these cells. CM-enriched fractions and cultures of fibroblasts were used for RNA isolation.

\section{Cell harvesting after $\mathrm{MS}$}

After undergoing MS or control treatment for $24 \mathrm{~h}$, cells were collected from CM-enriched cultures and from cultures of fibroblasts by trypsinisation. The proportion of $\mathrm{CM}$ in the cell cultures was determined by flow cytometric analysis of myosin expression as described before [35]. CM-enriched cell cultures, containing $54 \pm 5 \% \mathrm{CM}$, and fibroblast cultures, containing $95 \pm 2 \%$ non-myocytes, were used for RNA isolation.

\section{RNA isolation and labelling}

RNA was isolated applying an $\mathrm{RNeasy}^{\circledR}$ kit (Qiagen $\mathrm{GmbH}$, Hilden, Germany), according to the manufacturer's instructions. Ten to $14 \mu \mathrm{g}$ of total RNA from CMenriched fractions and 7.5 to $14 \mu \mathrm{g}$ of total RNA from fibroblast cultures was used for labelling. Per experiment, the quantities of total RNA from irradiated and control cells were equal. cDNA was synthesised using the Gibco BRL Superscript system (Invitrogen, Carlsbad CA, USA). Briefly, single stranded cDNA was synthesised using Superscript II reverse transcriptase and T7-oligo(dT)24 primers at $42^{\circ} \mathrm{C}$ for $1 \mathrm{~h}$. Double stranded cDNA was obtained by using DNA ligase, DNA polymerase I and RNAse $\mathrm{H}$ at $16^{\circ} \mathrm{C}$ for $2 \mathrm{~h}$, followed by T4 DNA polymerase at $16^{\circ} \mathrm{C}$ for $5 \mathrm{~min}$. After clean up with a phase lock gel (Qiagen), ds-cDNA was used for in vitro transcription (IVT). cDNA was transcribed using the BioArray HighYield ${ }^{\circledast}$ RNA transcript labelling kit (Enzo Lifesciences, Pharmingdale NY, USA) in the presence of biotin-labelled ribonucleotides, or using the MEGAScript $\mathrm{T} 7^{\circledast}$ kit (Ambion, Austin TX, USA), in the presence of biotinlabelled CTP and UTP. In each experiment, cDNAs from control cells and stressed cells were labelled simultaneously using the same labelling kit. After clean up with a RNeasy kit (Qiagen), the biotin-labelled IVT-RNA was fragmented in a buffer containing $40 \mathrm{mM}$ Tris-acetate $(\mathrm{pH}$ $8.1), 100 \mathrm{mM}$ potassium-acetate and $30 \mathrm{mM}$ magnesiumacetate, at $94^{\circ} \mathrm{C}$ for $35 \mathrm{~min}$.

\section{Hybridisation of IVT-RNA}

RG-U34A arrays (Affymetrix, Santa Clara CA, USA) were used, representing 7000 transcripts, of which 6399 had a unique LocusLink identification number (ID) at the time of data analysis. Biotin-labelled IVT-RNA was hybridised to the arrays at $45^{\circ} \mathrm{C}$ for $16 \mathrm{~h}$ according to the manufacturer's instructions. After hybridisation, the arrays were washed in a GeneChip Fluidics Station 400 with a nonstringent wash buffer at $25^{\circ} \mathrm{C}$ followed by a stringent wash buffer at $50^{\circ} \mathrm{C}$. After washing, the arrays were stained with a streptavidin-phycoerythrin complex. After staining, intensities were determined with a GeneChip scanner, controlled by GeneChip software (Affymetrix). The intensities were background corrected using gcrma [36] and normalized at the probe level by VSN [37].

\section{Data analysis}

For each stressor, three independent experiments were performed. To describe the signal of every PM probe, the following linear model was used: signal $\sim \mathrm{P}+\mathrm{T}+\mathrm{E}+\mathrm{TE}$, based on a method described before [15]. In our model, the symbols $\mathrm{P}, \mathrm{T}, \mathrm{E}$ and TE represent the effects of probe, treatment, experiment per stressor $(1,2$, or 3$)$ and the interaction between treatment and experiment, respectively. Subsequently, analysis of variance was applied on the treatment effect to determine the p-value for each 
probe-set. The p-values were corrected for multiple testing using the Benjamini Hochberg step-up procedure [38] which yielded q-values for the false discovery rates (FDR). The FDR level of control was set to 0.005 , equivalent to selecting genes with a q-value $<0.005$ (keeping up- and down-regulated genes separate by the sign of the treatment coefficient). Some genes are represented by more than one probe-set. A gene was determined to be differentially expressed when at least $50 \%$ of the probe-sets representing this gene showed significant up-regulation or down-regulation.

To determine significant proportions of differentially expressed genes within functional groups, the hypergeometric probability $\mathrm{P}$ was calculated. $\mathrm{P}<0.005$ was considered significant.

To determine the accuracy of the linear model that was used to describe PM signals, $\mathrm{R}^{2}$ was calculated for every probe-set, using the following standard formula: $\mathrm{R}^{2}=1$ $\Sigma \mathrm{R}_{\mathrm{i}}^{2} / \Sigma\left(\text { signal }_{\mathrm{i}} \text {-mean signal }\right)^{2}$, where $\mathrm{R}_{\mathrm{i}}=$ observed-fitted value for signal ${ }_{i}$ and mean signal $=$ the mean of observed signal $_{i}$.

To compare data on differentially expressed genes with microarray data in literature on species other than rat, RESOURCERER of the Institute of Genomic Research [39] was used.

\section{RNA isolation and semi-quantitative PCR}

Total RNA isolation, cDNA synthesis and semi-quantitative PCR were performed as described before [40]. In short, total RNA was isolated using an RNeasy ${ }^{\circledast}$ kit (Qiagen). Following DNAse (Amersham Pharmacia Biotech, Uppsala, Sweden) treatment, cDNA was synthesised using M-MLV reverse transcriptase (Life Technologies, Rockville, MD, USA) and oligo(dT) primers (Amersham). Semiquantitative PCR was performed, using the following primers and annealing temperatures: Tenascin $C$ sense: CGA CAG TTT TGT TAT CAG GAT CAG, Tenascin C antisense: GGC ACA TAA GTA ATC CGG AAA T, $60^{\circ} \mathrm{C}$; Biglycan sense: CAA CAA CCC TGT GCC CTA CT, Biglycan antisense: GGT GT GCT TCT TTG CTT CC, $65^{\circ} \mathrm{C}$. A competitive PCR was performed on GAPDH to correct for differences in cDNA concentrations. After agarose gel electrophoresis, intensities of PCR product bands were determined by Scion image analysis software (Scion Corporation, Frederick, MD, USA).

\section{Authors' contributions}

MB performed experiments, developed and analyzed functional groups of genes and prepared the manuscript. CGCW performed experiments, developed and analyzed functional groups of genes and participated in discussions. HV and AL participated in discussions and gave tex- tual advice. PS performed microarray normalization and data analysis. JW and LHFM participated in discussions. AAZ participated in discussions, suggested the assignment of genes to functional groups and gave textual advice. All authors read and approved the final manuscript.

\section{Additional material}

\begin{abstract}
Additional File 1
$q$-values of genes within functional groups Table 1. Excel-file containing the 25 functional groups of genes used in this study. Of every gene, Affymetrix probe-set IDs and q-values after the three types of stress are listed. Down-regulated genes are represented by a minus-sign in front of their q-value. q-values $<0.005$ are marked red (in case of up-regulation) and green (in case of down-regulation).

Click here for file

[http://www.biomedcentral.com/content/supplementary/14712164-6-6-S1.xls]
\end{abstract}

\section{Additional File 2}

General stress response genes Table 2. Excel file containing Affymetrix probe-set IDs, LocusLink ID and gene description of all probe-sets that showed an up-regulation or down-regulation after two or more stresses. Click here for file

[http://www.biomedcentral.com/content/supplementary/14712164-6-6-S2.xls]

\section{Acknowledgements}

This study was financially supported by the Netherlands Heart Foundation (grant no. 97.176), the Interuniversity Research Institute of Radiopathology and Radiation Protection (grant no. 9.0.13) and the European Union. The authors would like to thank Rebecca Esveldt-Van Lange, Cindy Schutte-Bart and Minka Bax for skilful technical support.

\section{References}

I. Tamamori-Adachi M, Ito H, Sumrejkanchanakij P, Adachi S, Hiroe M, Shimizu M, Kawauchi J, Sunamori M, Marumo F, Kitajima S, Ikeda MA: Critical role of cyclin DI nuclear import in cardiomyocyte proliferation. Circ Res 2003, 92:e I2-el 9.

2. Brilla CG, Maisch B: Regulation of the structural remodelling of the myocardium: from hypertrophy to heart failure. Eur Heart J 1994, I5 SuppI D:45-52.

3. Sadoshima J, Jahn L, Takahashi T, Kulik TJ, Izumo S: Molecular characterization of the stretch-induced adaptation of cultured cardiac cells. An in vitro model of load-induced cardiac hypertrophy. J Biol Chem 1992, 267:1055 I-10560.

4. Alenghat FJ, Ingber DE: Mechanotransduction: all signals point to cytoskeleton, matrix, and integrins. SCi STKE 2002, 2002(I I 9):PE6.Review.

5. Ruwhof $C$, van der Laarse A: Mechanical stress-induced cardiac hypertrophy: mechanisms and signal transduction pathways. Cardiovascular Research 2000, 47:23-37.

6. Adams MJ, Hardenbergh PH, Constine LS, Lipshultz SE: Radiationassociated cardiovascular disease. Crit Rev Oncol Hematol 2003, 45:55-75.

7. Dent P, Yacoub A, Contessa J, Caron R, Amorino G, Valerie K, Hagan MP, Grant S, Schmidt-Ullrich R: Stress and radiation-induced activation of multiple intracellular signaling pathways. Radiat Res 2003, I 59:283-300.

8. Di Leonardo A, Linke SP, Clarkin K, Wahl GM: DNA damage triggers a prolonged p53-dependent $G I$ arrest and long-term induction of Cip I in normal human fibroblasts. Genes Dev 1994, 8:2540-2551. 
9. Rodemann HP, Peterson HP, Schwenke K, von Wangenheim KH: Terminal differentiation of human fibroblasts is induced by radiation. Scanning Microscopy 1991, 5: I | 35- I | 42

10. Lampidis TJ, Weichselbaum RR, Little JB: Letter: Gamma-irradiation of mammalian beating heart cells in vitro. Effects on cellular function. Int J Radiat Biol Related Stud Phys Chem Med 1975, 28:99-102.

II. Petrovic D, Brown SM, Yatvin MB: Effects of adriamycin and irradiation on beating of rat heart muscle cells in culture. International Journal of Radiation Oncology, Biology, Physics 1977, 2:505-5I 3.

12. Ward JF: DNA damage as the cause of ionizing radiationinduced gene activation. Radiat Res 1994, I 38:S85-S88.

13. Kulms D, Schwarz T: Mechanisms of UV-induced signal transduction. J Dermatol 2002, 29:189-196.

14. van der Wees CG, Vreeswijk MP, Persoon M, van der Laarse A, van Zeeland AA, Mullenders LH: Deficient global genome repair of UV-induced cyclobutane pyrimidine dimers in terminally differentiated myocytes and proliferating fibroblasts from the rat heart. DNA Repair (Amst) 2003, 2: I297-I 308.

15. Chu TM, Weir B, Wolfinger R: A systematic statistical linear modeling approach to oligonucleotide array experiments. Math Biosci 2002, I 76:35-5I.

16. Jen KY, Cheung VG: Transcriptional response of lymphoblastoid cells to ionizing radiation. Genome Res 2003, I3:2092-2 100.

17. Gene Expression Omnibus (GEO) 2004 [http://
[ www.ncbi.nlm.nih.gov/geo/].

18. NettaffxTM 2004 [http://www.affymetrix.com/analysis/index.affx].

19. Bodyak N, Kang PM, Hiromura M, Sulijoadikusumo I, Horikoshi N, Khrapko K, Usheva A: Gene expression profiling of the aging mouse cardiac myocytes. Nucleic Acids Res 2002, 30:3788-3794.

20. Sesto A, Navarro M, Burslem F, Jorcano JL: Analysis of the ultraviolet $B$ response in primary human keratinocytes using oligonucleotide microarrays. Proc Natl Acad Sci U S A 2002 99:2965-2970.

21. Boerma M, Bart $\mathrm{Cl}$, Wondergem J: Effects of ionizing radiation on gene expression in cultured rat heart cells. Int J Radiat Biol 2002, 78:219-225.

22. Ruwhof $C$, van Wamel $A E$, van der Valk LJ, Schrier PI, van der Laarse $A$ : Direct, autocrine and paracrine effects of cyclic stretch on growth of myocytes and fibroblasts isolated from neonatal rat ventricles. Arch Physiol Biochem 2001, 109:10-17.

23. Heinloth AN, Shackelford RE, Innes CL, Bennett L, Li L, Amin RP, Sieber SO, Flores KG, Bushel PR, Paules RS: Identification of distinct and common gene expression changes after oxidative stress and gamma and ultraviolet radiation. Mol Carcinog 2003 , 37:65-82.

24. Brooks G, Poolman RA, McGill CJ, Li JM: Expression and activities of cyclins and cyclin-dependent kinases in developing rat ventricular myocytes. J Mol Cell Cardiol 1997, 29:226I-227I.

25. Garcia RH, Cabeza MP, Gallo A, Palacios L, Laguens RP: DNA content and expression of cell cycle proteins in caterpillar nuclei from fetal human cardiac myocytes. Virchows Arch 2002 , 440:45-49.

26. McBride WH, Iwamoto KS, Syljuasen R, Pervan M, Pajonk F: The role of the ubiquitin/proteasome system in cellular responses to radiation. Oncogene 2003, 22:5755-5773.

27. Shmeeda H, Petkova D, Barenholz Y: Cholesterol homeostasis in cultures of rat heart myocytes: relationship to cellular hypertrophy. Am J Physiol 1994, 267:HI689-HI697.

28. Kashiwagi Y, Haneda T, Osaki J, Miyata S, Kikuchi K: Mechanical stretch activates a pathway linked to mevalonate metabolism in cultured neonatal rat heart cells. Hypertens Res 1998 , 2l:109-II9.

29. Okumura K, Kondo J, Yoshino M, Ishikawa K, Asano H, Hashimoto $H$, Ito $T$ : Enalapril reduces the enhanced I,2-diacylglycerol content and RNA synthesis in spontaneously hypertensive rat hearts before established hypertension. Mol Cell Biochem 1992, I | 2: 15-21.

30. Bastiaanse EM, Hold KM, van der Laarse A: The effect of membrane cholesterol content on ion transport processes in plasma membranes. Cardiovasc Res 1997, 33:272-283.

31. Yechiel E, Henis YI, Barenholz Y: Aging of rat heart fibroblasts: relationship between lipid composition, membrane organization and biological properties. Biochim Biophys Acta 1986, 859:95-104
32. Yechiel E, Barenholz Y, Henis YI: Lateral mobility and organization of phospholipids and proteins in rat myocyte membranes. Effects of aging and manipulation of lipid composition. J Biol Chem 1985, 260:9|32-9136.

33. Yechiel E, Barenholz Y: Relationships between membrane lipid composition and biological properties of rat myocytes. Effects of aging and manipulation of lipid composition. J Biol Chem 1985, 260:9123-9131.

34. van der Laarse A, Hollaar L, Kokshoorn LJ, Witteveen SA: The activity of cardio-specific isoenzymes of creatine phosphokinase and lactate dehydrogenase in monolayer cultures of neonatal rat heart cells. I Mol Cell Cardiol 1979, I I:50 I-5 I0.

35. Boerma M, van der Wees CG, Wondergem J, van der Laarse A, Persoon M, van Zeeland AA, Mullenders LH: Separation of neonatal rat ventricular myocytes and non-myocytes by centrifugal elutriation. Pflugers Arch 2002, 444:452-456.

36. Wu Z, Irizarry RA: Preprocessing of oligonucleotide array data. Nat Biotechnol 2004, 22:656-658.

37. Huber W, Von Heydebreck A, Sultmann H, Poustka A, Vingron M: Variance stabilization applied to microarray data calibration and to the quantification of differential expression. Bioinformatics 2002, I 8 Suppl I:S96-SI04

38. Benjamini $Y$, Hochberg $Y$ : Controlling the false discovery rate: a practical and powerful approach to multiple testing. J Roy Statist Soc B 2004, 64:289-300.

39. RESOURCERER 2004 [http://www.tigr.org/tigr-scripts/magic/ rl.pl].

40. Boerma M, Schutte-Bart Cl, Wedekind LE, Beekhuizen H, Wondergem J: Effects of multiple doses of ionizing radiation on cytokine expression in rat and human cells. Int J Radiat Biol 2003, 79:889-896.

Publish with Biomed Central and every scientist can read your work free of charge

"BioMed Central will be the most significant development for disseminating the results of biomedical research in our lifetime. "

Sir Paul Nurse, Cancer Research UK

Your research papers will be:

- available free of charge to the entire biomedical community

- peer reviewed and published immediately upon acceptance

- cited in PubMed and archived on PubMed Central

- yours - you keep the copyright
BioMedcentral 